Rev. Int. Contam. Ambie. 35 (2) 421-434, 2019

DOI: $10.20937 /$ RICA.2019.35.02.13

\title{
ESTIMACIÓN DE LA ISLA DE CALOR URBANA EN MEDELLÍN, COLOMBIA
}

\author{
(Estimation of the urban heat island in Medellin, Colombia)
}

\author{
Engelberth SOTO-ESTRADA
}

Programa de Ingeniería Civil, Universidad EIA, Envigado, Colombia

Correo electrónico: engelberth.soto@eia.edu.co

(Recibido diciembre 2017; aceptado junio 2018)

Palabras clave: temperatura superficial, Landsat, NDVI

\section{RESUMEN}

Isla de calor urbana (ICU) es un término utilizado para describir el incremento de temperatura, superficial y atmosférica, relacionado con el desarrollo urbano. Las islas de calor incrementan el consumo energético, la contaminación atmosférica, la contaminación térmica de cuerpos de agua y, en general, disminuyen de la calidad de vida de la población. Debido a ello, la mitigación de la ICU y el desarrollo de estrategias de adaptación son prioridades en diversas ciudades del planeta. A pesar de que este efecto puede percibirse en el Valle de Aburrá, lugar donde se encuentra la ciudad de Medellín, ha sido escasamente estudiado y hasta el momento no existen medidas concretas de escala metropolitana para mitigarlo. En este artículo se presenta la estimación de la ICU en la región metropolitana del Valle de Aburrá. La ICU se calculó utilizando imágenes Landsat TM, ETM+ y OLI/TIRS, adquiridas entre los años 1986 y 2016. Los resultados mostraron que, en promedio, la superficie de la zona urbana fue $4.81^{\circ} \mathrm{C}$ más caliente que las coberturas del área rural que la rodea, concentrándose este efecto en los barrios de la zona central del valle.

Key words: surface temperature, Landsat, NDVI

\begin{abstract}
The urban heat island (UHI) effect describes the phenomenon whereby cities are generally warmer than surrounding rural areas. UHI increases energy consumption, air and water pollution, and, in general, reduces the urban life quality. Consequently, UHI mitigation and adaptation is a priority in many cities around the world. Although the UHI effect can be perceived in the Aburra Valley, where the city of Medellin is located, it has been studied superficially and so far there are no prevention or mitigation measures at the regional scale. This paper presents an estimation of the UHI in the metropolitan area of the Aburra Valley. The UHI was estimated using Landsat TM, ETM+ and OLI/TIRS images acquired between 1986 and 2016. Results showed that the average surface temperature in the urban area was $4.81{ }^{\circ} \mathrm{C}$ warmer than in the rural surroundings.
\end{abstract}




\section{INTRODUCCIÓN}

El término isla de calor urbana (ICU) se utiliza para describir la tendencia de áreas urbanas a experimentar incrementos de temperatura, en comparación con las zonas suburbanas o rurales que las rodean (Landsberg 1981). Estas anomalías se clasifican en islas de calor atmosféricas (capa de dosel y capa de perímetro) e islas de calor superficiales (Voogt 2008).

Las islas de calor se asocian con el desarrollo urbano. El crecimiento de las ciudades origina el reemplazo de las coberturas naturales con superficies artificiales, lo que reduce la permeabilidad del suelo y con ello el proceso de evapotranspiración que favorece la refrigeración del entorno (NASA 2015). La intensidad de las ICU depende de múltiples factores, entre ellos densidad constructiva, orientación de las edificaciones, comportamiento térmico de los materiales utilizados y disponibilidad y configuración de los espacios abiertos y áreas verdes (Asimakopoulos et al. 2001, Gartland 2008, Erell et al. 2011, Aleksandrowicz et al. 2017).

La evidencia sugiere que las ICU provocan un impacto negativo en los ecosistemas y el clima locales (Sarrat et al. 2006, Corumluoglu y Asri 2015, Qunfang y Yuqi 2015, Qaid et al. 2016), además de que aumentan la demanda energética (Akbari et al. 2001, Ihara et al. 2008, Kolokothoni et al. 2012, Arifwidodo y Chandrasiri 2015). Adicionalmente, las ICU incrementan la ocurrencia de enfermedades relacionadas con las altas temperaturas, como dificultades respiratorias e insolación (Bai et al. 2014), así como otras cuyos vectores se ven favorecidos con las nuevas condiciones climáticas (Vieira-Araujo et al. 2015). En general, las ICU disminuyen la calidad de vida de la población y su productividad. Debido a ello, la mitigación de las ICU es una prioridad en diversas ciudades alrededor del mundo como Nueva York (NYSERDA 2006), Toronto (City of Toronto 2008) y múltiples poblaciones japonesas (Japan for Sustainability 2008), por mencionar algunos ejemplos.

Los efectos de las ICU pueden ser mayores en los países en vías de desarrollo. Scott (2006) y CampbellLendrum y Corvalán (2007) señalan que la expansión acelerada de las ciudades en estos países, la cual no es acorde con su crecimiento económico, incrementará el número de habitantes en condiciones de vulnerabilidad ante ICU y olas de calor. En el grupo de personas vulnerables se incluyen los habitantes de viviendas construidas con materiales que no proporcionan aislamiento térmico, como láminas y otros materiales ligeros, y aquellos con acceso limitado a sistemas de refrigeración y atención médica.
El área metropolitana localizada en el Valle de Aburrá (conocida localmente como AMVA, aunque las siglas también se asocian con la autoridad ambiental regional), en el departamento colombiano de Antioquia, no está exenta de esta problemática. Los habitantes perciben un aumento en la temperatura de la ciudad, lo que es congruente con los registros de estaciones meteorológicas. La autoridad ambiental del AMVA y la Universidad Nacional de Colombia (AMVA-UNAL 2013), analizaron los registros mensuales de temperatura atmosférica correspondientes al periodo 1942-2009. De acuerdo con la información de la estación Aeropuerto Olaya Herrera, la cual se localiza en la zona central del valle, a lo largo de este periodo se ha presentado un incremento promedio de $0.7^{\circ} \mathrm{C}$ por década para los registros de temperatura mínima y $0.2^{\circ} \mathrm{C}$ por década para temperatura media.

El Valle de Aburrá posee un área de $1152 \mathrm{~km}^{2}$ (Aristizábal y Yokota 2008), que hacen parte de la cuenca del río del mismo nombre. Tiene una longitud aproximada de $65 \mathrm{~km}$ y un ancho máximo en su parte plana de alrededor de $10 \mathrm{~km}$, por lo que se cataloga como un valle estrecho. Su topografía varía de 1300 a 2800 msnm (AMVA 2010). La precipitación de esta zona está controlada principalmente por los vientos alisios, cuyo recorrido es modificado por la topografía del valle. Los vientos dominantes se registran en la dirección noreste, con velocidades promedio de aproximadamente $2 \mathrm{~m} / \mathrm{s}$ (Correa et al. 2009). Se presentan dos estaciones húmedas alrededor de abril y octubre, con precipitaciones que van de $1400 \mathrm{~mm}$ en el norte del valle hasta unos $3000 \mathrm{~mm}$ en la parte sur (Hermelin 2007). Las temperaturas medias anuales varían en función de la altura y del ciclo diurnonocturno, ya que la localización del valle, alrededor de $6^{\circ} 30^{\prime}$ al norte del ecuador, impide variaciones térmicas estacionales (Pérez 1993). En la parte baja del valle, a lo largo del corredor fluvial, se registran temperaturas medias anuales de 20 a $24^{\circ} \mathrm{C}$, en tanto que en la parte alta valores del orden de los $17^{\circ} \mathrm{C}$ (UNAL 2005). La humedad relativa del aire fluctúa durante el año entre 63 y $73 \%$, siendo mayor en la época lluviosa del segundo semestre (IDEAM 2010).

En el Valle de Aburrá se localiza un área urbana compuesta por 10 municipios. Medellín es el mayor de ellos, tanto en extensión como en población. En esta región viven alrededor de 3.7 millones de personas en una superficie aproximada de $200 \mathrm{~km}^{2}$ (AMVA 2017).

En esta investigación se estimó la isla de calor superficial (ICS) del AMVA utilizando 15 imágenes satelitales Landsat 5, 7 y 8, sensores TM (Thematic Mapper), ETM+ (Enhanced Thematic Mapper Plus) 
y OLI/TIRS (Operational Land Imager/Thermal Infrared Sensor), adquiridas durante el periodo 1986-2016. Adicionalmente, se calculó la densidad de vegetación mediante el índice de vegetación de diferencia normalizada (NDVI, por sus siglas en inglés). La relación entre la presencia de vegetación y la intensidad de la ICU ha sido extensamente documentada (Susca et al. 2011, Watkins y Kolokotroni 2012, Kieron y Hutchings 2013, Buyadi et al. 2014, US-EPA 2016), razón por la cual se consideró a la presencia de vegetación como variable de interés. Con esta información se identificaron zonas urbanas críticas, lo que se espera contribuya a la priorización de las estrategias de mitigación y adaptación en la ciudad. En este artículo se presenta la descripción de la ICS en el Valle de Aburrá.

\section{MATERIALES Y MÉTODOS}

El primer paso consistió en identificar las imágenes Landsat con cobertura en el área de estudio (path 9, row 56) en la base de datos del Servicio Geológico de Estados Unidos (USGS, por sus siglas en inglés) (USGS-EarthExplorer 2017). Se descargaron más de 30 imágenes, de las cuales se seleccionaron 15 . Para la selección se consideró que la zona de estudio estuviera libre de nubosidad, así como de los errores de adquisición relacionados con la falla del corrector de escaneo lineal (SLC, por sus siglas en inglés) que afecta a algunas imágenes Landsat 7 . El cuadro I contiene la fecha de adquisición y el tipo de sensor de las imágenes utilizadas.

\section{CUADRO I. IMÁGENES LANDSAT ANALIZADAS}

\begin{tabular}{cccc}
\hline Satélite & $\begin{array}{c}\text { Fecha de } \\
\text { adquisición }\end{array}$ & Satélite & $\begin{array}{c}\text { Fecha de } \\
\text { adquisición }\end{array}$ \\
\hline Landsat 5 & $8-09-1986$ & Landsat 7 & $24-05-2014$ \\
Landsat 7 & $21-08-2000$ & Landsat 8 & $20-08-2014$ \\
Landsat 7 & $18-04-2001$ & Landsat 8 & $11-01-2015$ \\
Landsat 7 & $2-01-2003$ & Landsat 7 & $16-09-2015$ \\
Landsat 5 & $16-06-2008$ & Landsat 8 & $29-12-2015$ \\
Landsat 7 & $7-09-2012$ & Landsat 8 & $22-06-2016$ \\
Landsat 7 & $8-07-2013$ & Landsat 7 & $21-11-2016$ \\
Landsat 7 & $13-11-2013$ & & \\
\hline
\end{tabular}

Todas las imágenes se descargaron con un nivel de procesamiento 1 (L1T y L1TP). Los productos de este nivel se encuentran calibrados radiométricamente y ortorrectificados mediante puntos de control y modelos digitales de elevación para corregir el desplazamiento debido al relieve. Adicionalmente, las bandas térmicas de los sensores ETM+ y OLI/ TIRS han sido remuestreadas con una resolución de $30 \mathrm{~m}$. Estos son los productos Landsat de mayor calidad, por lo que son recomendados para el análisis de series de tiempo a nivel de pixel (USGS 2017a).

La corrección atmosférica se realizó con las herramientas Quick Atmospheric Correction (QUAC) y Thermal Atmospheric Correction del programa ENVI 5.3. QUAC es un método diseñado para las regiones del espectro visible, cercano al infrarrojo e infrarrojo de onda corta (VNIR-SWIR, 400-2500 $\mathrm{nm})$. Este algoritmo determina los parámetros de compensación analizando los pixeles de la escena. $\mathrm{Su}$ uso es recomendado en imágenes con al menos 10 tipos de coberturas y suficientes pixeles oscuros para la estimación de la línea de base espectral. Por su parte, el algoritmo para corrección de bandas térmicas (8000-14 $000 \mathrm{~nm}$ ) asume que la atmósfera es uniforme. Basa su funcionamiento en la longitud de onda que muestra la mayor temperatura de brillo en la imagen (Harris Geospatial Solutions 2017).

La temperatura superficial (TS) se determinó mediante el cálculo de la temperatura de brillo en el tope de la atmósfera (TOA, por sus siglas en inglés) y la emisividad. La temperatura de brillo o temperatura radiante se estimó utilizando la ecuación 1 , indicada por la Administración Nacional de la Aeronáutica y del Espacio de los Estados Unidos de América (NASA 2007), la cual se aplicó a las bandas térmicas mostradas en el cuadro II.

$T_{r}=\frac{K 2}{\ln \left(\frac{K 1}{L \lambda}+1\right)}$

donde $T_{r}$ es la temperatura radiante en grados Kelvin; $K 1$ la constante de calibración 1 (cuadro III); $K 2$ la constante de calibración 2 (cuadro III), y $L_{\lambda}$ la irradiación espectral en la apertura del sensor en watts $/\left(\mathrm{m}^{2} \times\right.$ ster $\left.\times \mu \mathrm{m}\right)$.

La emisividad se estimó a partir del índice NDVI utilizando un método de umbrales (Sobrino et al. 1999), como se explica a continuación:

a. NDVI $<0.2$ se consideró como suelo sin vegetación en zona urbana; a estos pixeles se les asignó un valor de emisividad de 0.92 (Sobrino et al. 2012).

b. NDVI $>0.5$ se consideró como superficie cubierta totalmente con vegetación; estos pixeles fueron calificados con un valor de 0.99 (Sobrino et al. 2004). 
CUADRO II. BANDAS TÉRMICAS UTILIZADAS

\begin{tabular}{llcc}
\hline Satélite & Banda & Ancho de banda $(\mu \mathrm{m})$ & Resolución $(\mathrm{m})$ \\
\hline Landsat 5 & 6- Térmica & $10.40-12.50$ & 30 \\
Landsat 7 & 62- Térmica de alta resolución radiométrica & $10.40-12.50$ & $60(30)^{*}$ \\
Landsat 8 & 10- Térmica infrarroja (TIRS) 1 & $10.60-11.19$ & $100(30)^{*}$ \\
\hline
\end{tabular}

Fuente: construido con información de USGS 2017b. *Las imágenes se entregan remuestreadas con $30 \mathrm{~m}$ de resolución

CUADRO III. CONSTANTES DE CALIBRACIÓN K1 Y K2

\begin{tabular}{lcc}
\hline Satélite & $\mathrm{K} 1$ & $\mathrm{~K} 2$ \\
& $\left(\right.$ watts $/\left[\mathrm{m}^{2} \times\right.$ ster $\left.\left.\times \mu \mathrm{m}\right]\right)$ & $\left(\right.$ watts $/\left[\mathrm{m}^{2} \times\right.$ ster $\left.\left.\times \mu \mathrm{m}\right]\right)$ \\
\hline Landsat 5 & 607.76 & 1260.56 \\
Landsat 7 & 666.09 & 1282.71 \\
Landsat 8 & De metadatos & De metadatos \\
\hline
\end{tabular}

Fuente: construido con información de NASA 2007 y USGS 2016

c. $0.2 \leq \mathrm{NDVI} \leq 0.5$ se supuso correspondiente a pixeles con cobertura mixta; la emisividad de estas zonas se calculó mediante la ecuación 2 (Sobrino et al. 2004):

$\varepsilon=0.004 P_{V}+0.986$

donde $\varepsilon$ es la emisividad en pixeles con cobertura mixta y $P_{v}$ la proporción de vegetación, la cual fue estimada mediante la ecuación 3 (Carlson y Ripley 1997):

$P_{V}=\left[\frac{N D V I-N D V I_{\min }}{N D V I_{\max }-N D V I_{\min }}\right]^{2}$

donde $N D V I_{\min }=0.2$ y $N D V I_{\max }=0.5$.

Por su parte, NDVI se calculó con la ecuación 4 (Rouse et al. 1974):

$N D V I=\frac{(N I R-R E D)}{(N I R+R E D)}$

donde NIR es la banda cercana al infrarrojo (banda 4 para los sensores TM y ETM+ y 5 para el sensor OLI/ TIRS); RED la banda roja (banda 3 para los sensores TM y ETM+ y 4 para el sensor OLI/TIRS). NDVI informa sobre la cantidad y la calidad de la vegetación utilizando la reflexión de la clorofila en la región espectral del infrarrojo cercano (700-1100 $\mathrm{nm}$ ). NDVI produce valores entre -1 y 1 para cada pixel.
Valores cercanos a $1(0.8$ a 0.9$)$ indican la mayor densidad posible de hojas verdes, en tanto que los valores próximos a -1 significan ausencia de vegetación (NASA 2012).

La temperatura de superficie se estimó con base en la ecuación 5 (Artis y Carnahan 1982):

$T_{s}=\frac{T_{r}}{1+\left(\frac{\lambda T_{r}}{\rho}\right) \ln \varepsilon}$

donde $T_{\mathrm{s}}$ es la temperatura superficial en grados Kelvin; $T_{r}$ es la temperatura radiante en grados Kelvin; $\lambda$ el valor medio del ancho de banda $(11.5 \mu \mathrm{m}$ para los sensores TM y ETM+ y $10.8 \mu \mathrm{m}$ para el sensor OLI/TIRS) $\rho=1.438 \times 10^{-2} \mathrm{~m} \mathrm{~K}$ con base en las constantes de Boltzmann, Planck y la velocidad de la luz, y $\varepsilon$ es la emisividad. Finalmente, la temperatura de superficie se transformó a grados centígrados utilizando la relación $\mathrm{C}=\mathrm{K}-273.15$.

El análisis de temperatura superficial y densidad de vegetación se basó en la identificación de tendencias de cambio a lo largo del periodo de estudio utilizando una división por barrios. Se propuso esta unidad de análisis con el supuesto de que los barrios presentan una tipología urbana característica, la cual podría relacionarse con su temperatura superficial y, posteriormente, en investigaciones futuras, con su temperatura atmosférica. Para cada barrio se estimó la temperatura superficial promedio. Lo mismo se realizó con los valores de NDVI, calculándose posteriormente el porcentaje de cambio de vegetación a lo largo del periodo de estudio. Las tendencias de cambio se analizaron mediante una prueba de hipótesis t sobre los parámetros de la recta de regresión.

\section{DISCUSIÓN DE RESULTADOS}

La figura 1 muestra un ejemplo de la estimación de TS y densidad de vegetación, en este caso, la correspondiente al 21 de noviembre de 2016. En 

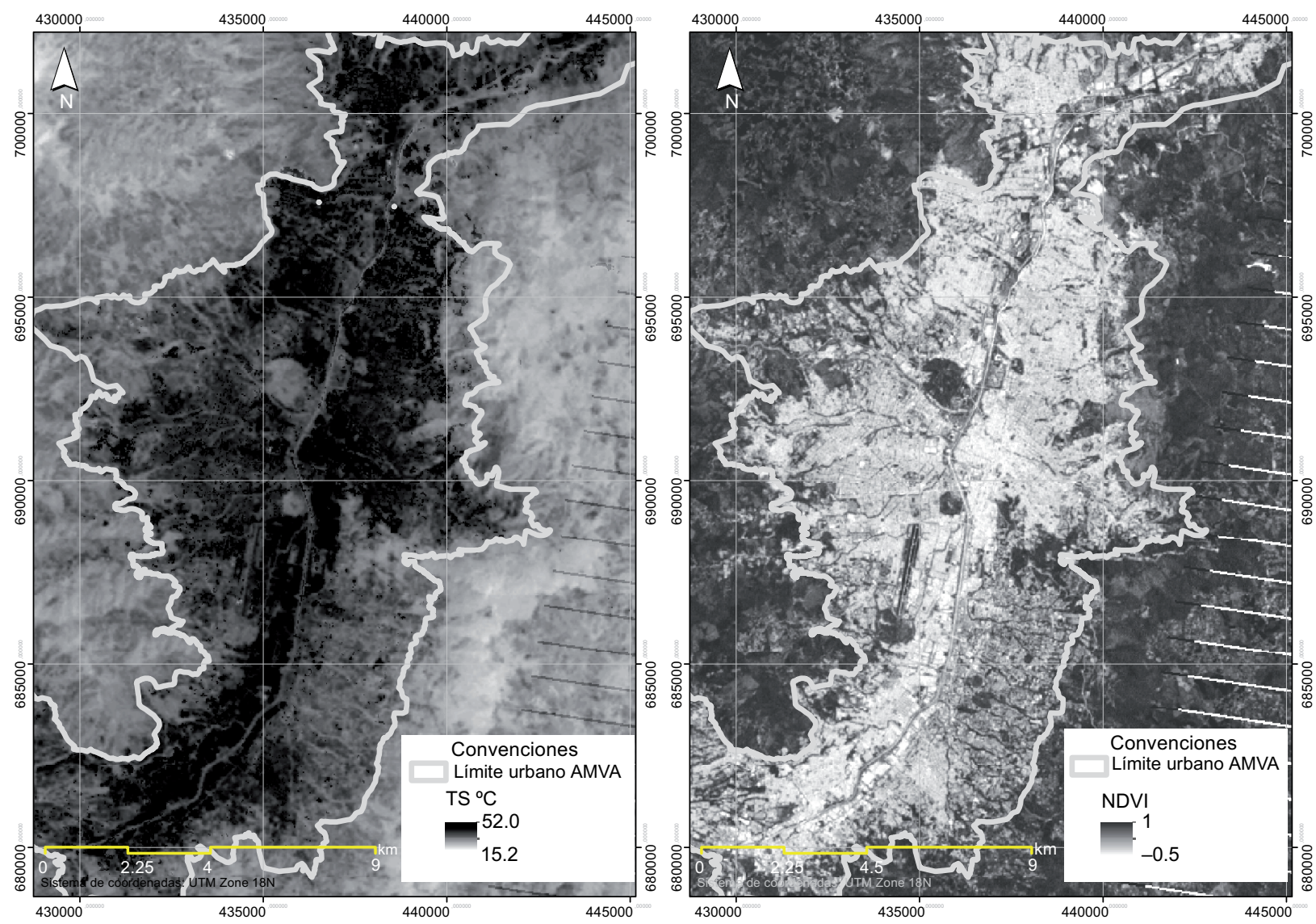

Fig. 1. Temperatura superficial (izquierda) y densidad de vegetación (derecha) en el área de estudio el 21 de noviembre de 2016

estas imágenes se pueden apreciar el área urbana localizada en el Valle de Aburrá (delimitada con el polígono blanco) y el río del mismo nombre que corre por la parte central del valle con una dirección predominante suroeste-noreste (línea clara diagonal en la imagen de temperatura y oscura en la de NDVI).

Los valores de TS de la figura 1 corresponden a las condiciones predominantes a las 10:00 a.m. del día mencionado. Los satélites Landsat se encuentran en órbitas heliosincrónicas, lo que significa que siempre pasan a la misma hora por un determinado lugar. Los satélites Landsat 7 cruzan el ecuador a las 10 a.m. \pm 15 minutos, en tanto que los Landsat 8 a las 10:11 a.m. \pm 15 minutos; a esta hora se busca obtener imágenes con la máxima iluminación y el mínimo vapor de agua en la atmósfera (USGS 2017c). En estas condiciones, el 21 de noviembre de 2016 se registraron valores de TS entre 15.2 y $52.0^{\circ} \mathrm{C}$ en el área urbana del Valle de Aburrá, con una TS promedio de $27.8^{\circ} \mathrm{C}$.

Para estimar la intensidad de la isla de calor (IIC) se calcularon los valores de TS del área rural que circunda la zona urbana. Se eligió una franja de 1 km a partir del límite urbano (Fig. 2). El 21 de noviembre de 2016 se registraron valores de TS entre 13.1 y $39.9^{\circ} \mathrm{C}$ en esta franja, con una TS promedio de $21.7^{\circ} \mathrm{C}$. Por tanto, la IIC fue de $6.1^{\circ} \mathrm{C}$ alrededor de las 10 a.m. del día mencionado.

En el cuadro IV se muestran los valores de temperatura superficial mínimo, máximo y promedio, así como el IIC para cada uno de los días examinados.

Considerando todos los mapas calculados, en la zona rural que rodea el AMVA se estimaron valores de TS de 0.83 a $43.50{ }^{\circ} \mathrm{C}$, mientras que en la región urbana de 1.30 a $52.80{ }^{\circ} \mathrm{C}$. En promedio, el área urbana registró una TS de $25.85^{\circ} \mathrm{C}$ y el área rural contigua (hasta $1 \mathrm{~km}$ de distancia desde el borde urbano) $21.04{ }^{\circ} \mathrm{C}$, por lo que la intensidad de la isla de calor promedio, es decir, la diferencia entre la temperatura superficial promedio de la zona habitada y la de las zonas rurales adyacentes, fue de $4.81{ }^{\circ} \mathrm{C}$.

Las imágenes Landsat han sido ampliamente utilizadas en el estudio de las ICU. No obstante, el análisis de regiones como el Valle de Aburrá supone retos 


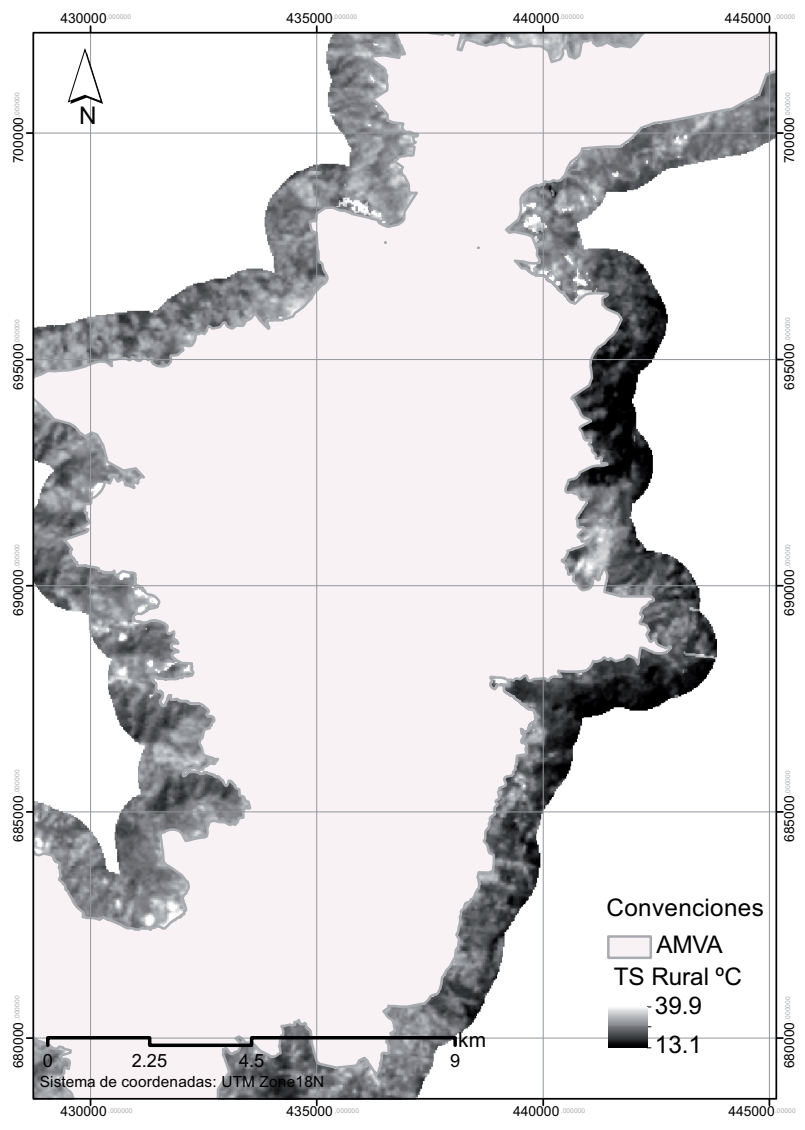

Fig. 2. Temperatura superficial en una franja de $1 \mathrm{~km}$ a partir de la zona urbana, valores del 21 de noviembre de 2016 adicionales. A diferencia de otros sitios como Las Vegas (Black 2013) o Nueva York (NASA 2006), donde la topografía del terreno es predominantemente plana, en el Valle de Aburrá existe una diferencia de alrededor de $1500 \mathrm{~m}$ entre sus partes altas y bajas, por lo que el relieve del terreno tiene influencia sobre la intensidad y distribución de la ICS. A las 10 de la mañana, la ladera occidental del valle ha recibido más radiación solar que la ladera oriental. Aunado a ello, la elevación del terreno, la exposición a los vientos, la humedad y la presencia de vegetación provocan que, en general, las partes altas del valle sean más frías que la parte baja. Por esta razón, para comparar la TS promedio de la zona urbana con la registrada en el área rural se utilizó una franja de $1 \mathrm{~km}$ a partir del límite urbano, de manera que el efecto de los aspectos mencionados fuera limitado y los valores comparables.

La influencia del relieve del terreno y la ocupación urbana en la TS del valle se puede apreciar en la figura 3, en la cual se muestran los perfiles de TS obtenidos con cada una de las imágenes satelitales utilizadas. Los perfiles fueron extraídos de los mapas de temperatura superficial empleando un eje arbitrario este-oeste que pasa por el centro de la zona de estudio. La figura 3 incluye el perfil de terreno (línea gruesa) a lo largo del mismo eje y una indicación de la ubicación de la zona urbana. En el eje horizontal se incluyeron coordenadas de referencia en el sistema UTM Zone $18 \mathrm{~N}$.

CUADRO IV. TEMPERATURA SUPERFICIAL E INTENSIDAD DE LA ISLA DE CALOR A LO LARGO DEL PERIODO DE ESTUDIO

\begin{tabular}{|c|c|c|c|c|c|c|c|c|c|}
\hline \multirow{2}{*}{ Fecha } & \multicolumn{3}{|c|}{$\begin{array}{c}\text { Urbano } \\
\left({ }^{\circ} \mathrm{C}\right)\end{array}$} & \multicolumn{3}{|c|}{$\begin{array}{l}\text { Rural } \\
\left({ }^{\circ} \mathrm{C}\right)\end{array}$} & \multicolumn{3}{|c|}{$\begin{array}{l}\text { IIC } \\
\left({ }^{\circ} \mathrm{C}\right)\end{array}$} \\
\hline & Min & Max & Prom. & Min & Max & Prom. & Min & Max & Prom. \\
\hline 8-09-1986 & 15.11 & 41.00 & 25.46 & 13.77 & 35.96 & 22.05 & 1.34 & 5.04 & 3.41 \\
\hline $21-08-2000$ & 1.30 & 39.00 & 19.17 & 6.45 & 30.85 & 12.94 & -5.15 & 8.15 & 6.23 \\
\hline $18-04-2001$ & 4.90 & 42.51 & 24.34 & 3.40 & 33.15 & 20.01 & 1.50 & 9.36 & 4.33 \\
\hline $2-01-2003$ & 9.26 & 43.04 & 26.97 & 9.22 & 39.82 & 22.70 & 0.04 & 3.22 & 4.27 \\
\hline $16-06-2008$ & 6.88 & 37.54 & 23.48 & 4.31 & 32.35 & 19.82 & 2.57 & 5.19 & 3.66 \\
\hline 7-09-2012 & 17.71 & 52.80 & 30.19 & 15.91 & 42.51 & 24.22 & 1.80 & 10.29 & 5.97 \\
\hline $8-07-2013$ & 8.37 & 46.71 & 26.49 & 4.25 & 37.08 & 21.63 & 4.12 & 9.63 & 4.86 \\
\hline $13-11-2013$ & 2.58 & 36.52 & 19.12 & 0.83 & 31.14 & 15.11 & 1.75 & 5.38 & 4.01 \\
\hline 24-05-2014 & 11.14 & 46.97 & 25.68 & 8.56 & 38.18 & 21.40 & 2.58 & 8.79 & 4.28 \\
\hline 20-08-2014 & 9.09 & 40.55 & 26.91 & 6.84 & 34.16 & 20.97 & 2.25 & 6.39 & 5.94 \\
\hline $11-01-2015$ & 10.44 & 44.35 & 28.39 & 6.92 & 43.50 & 23.83 & 3.52 & 0.85 & 4.56 \\
\hline $16-09-2015$ & 4.64 & 50.29 & 29.68 & 3.96 & 40.09 & 23.50 & 0.68 & 10.20 & 6.18 \\
\hline $29-12-2015$ & 11.90 & 43.57 & 28.27 & 9.81 & 39.34 & 24.19 & 2.09 & 4.23 & 4.08 \\
\hline 22-06-2016 & 7.43 & 43.20 & 25.90 & 6.68 & 36.22 & 21.58 & 0.75 & 6.98 & 4.32 \\
\hline 21-11-2016 & 15.20 & 52.05 & 27.76 & 13.14 & 39.90 & 21.67 & 2.06 & 12.15 & 6.09 \\
\hline Promedio & 9.06 & 44.01 & 25.85 & 7.60 & 36.95 & 21.04 & 1.46 & 7.06 & 4.81 \\
\hline
\end{tabular}


En la figura 3 se aprecia el comportamiento típico de las ICU, que responden, primordialmente, a la densidad de ocupación. En la parte central de la gráfica se observa una disminución de TS. Esta zona corresponde al río Aburrá, que se ubica en el punto más bajo del perfil de terreno del valle (cerca de la coordenada 436000 en la Fig. 3).

La figura 3 proporciona un ejemplo del perfil transversal de la ICS en el Valle de Aburrá. Se puede apreciar que las TS estimadas a lo largo de los 30 años de análisis fluctúan en una franja (diferencia entre el valor mínimo y el máximo para el mismo sitio) de 10 (lado izquierdo de la gráfica) a $20{ }^{\circ} \mathrm{C}$ (lado derecho). Estas diferencias obedecen, como se mencionó anteriormente, a que la radiación solar no ha incidido de manera homogénea en el valle a la hora en que se adquieren las imágenes Landsat. A las 10 de la mañana la ladera occidental (lado izquierdo en la Fig. 3) ha recibido más radiación solar que la ladera oriental.

Por otra parte, la variación temporal de TS se debe a diversos factores, como las condiciones climáticas y los cambios en la superficie del terreno. Gedzelman et al. (2003) señalan que las islas de calor son más intensas con cielos despejados y cuando el viento y la lluvia están ausentes, y se reducen, consecuentemente, en condiciones opuestas.
El calor específico del suelo varía notablemente según su contenido de humedad (UPM 2009). Los suelos húmedos se calientan más lentamente que los secos, pero mantienen más el calor reduciendo las variaciones de temperatura. Por otra parte, la conductividad térmica del suelo depende del acomodo de sus partículas y del contenido de humedad. Los suelos húmedos conducen mejor el calor que los secos. La presencia de materiales artificiales también modifica la cantidad de radiación reflejada (albedo). El albedo del suelo (desnudo) puede encontrarse entre 0.05 y 0.20 , en tanto que el de zonas urbanas entre 0.1 y 0.27 (Jacobson 1999).

Los factores climáticos con incidencia en la TS como la lluvia, el viento o la presencia de nubosidad no se distribuyen de manera homogénea en el Valle de Aburrá, como se explicó en la sección de introducción. No se cuenta con información detallada sobre la distribución de estos factores en la zona de estudio para los distintos momentos de análisis, por lo que los valores de TS estimados no pueden interpretarse completamente. En la figura 4 se presentan los valores de TS promedio para cada imagen satelital. La prueba de hipótesis en la recta de regresión indicó que probablemente no existe tendencia lineal en los valores de TS promedio analizados $\left(t_{0}=0.00027973\right.$ $\left.<t_{\text {crit }}=2.17881283\right)$.

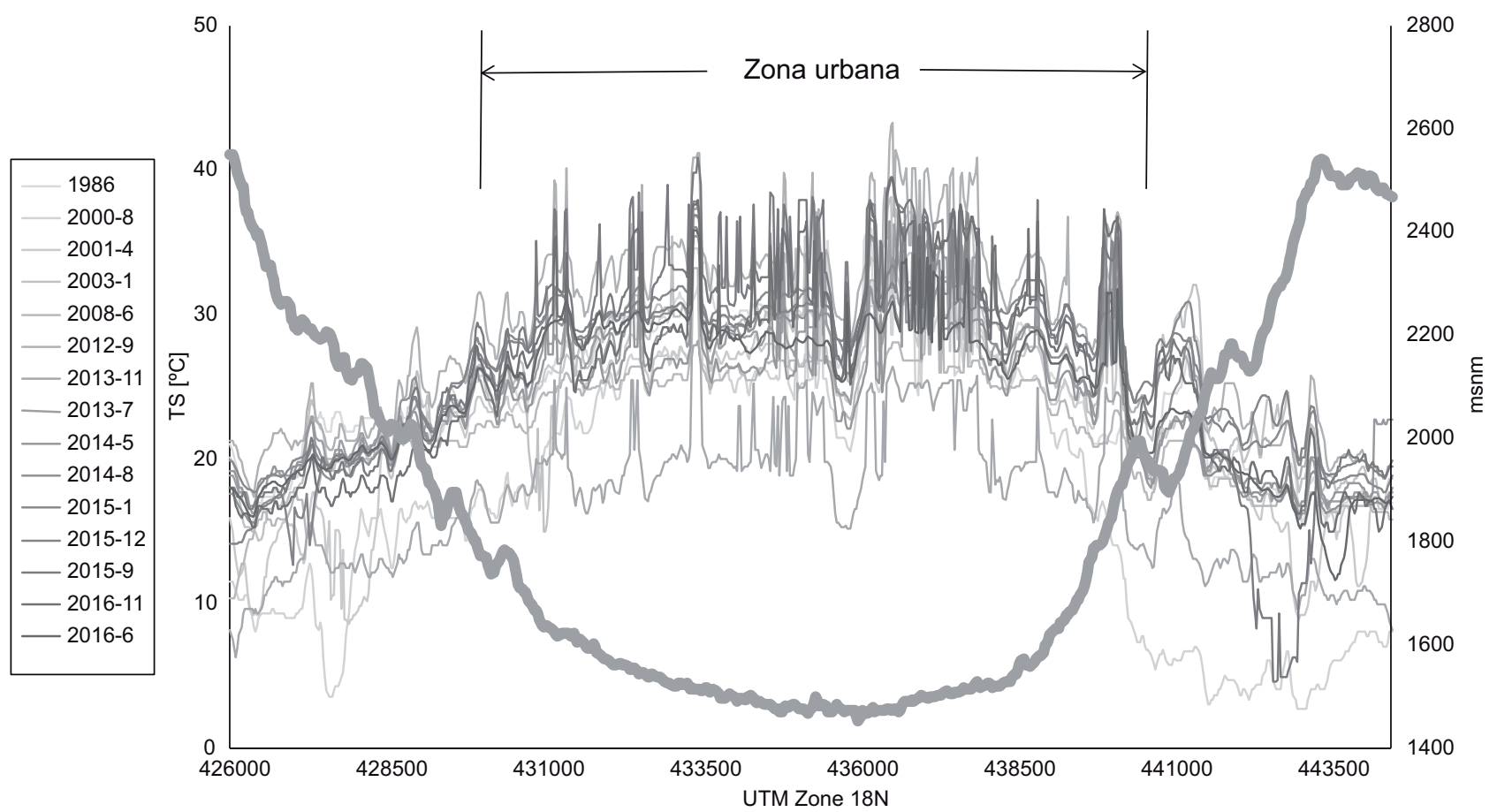

Fig. 3. Perfiles de temperatura superficial y altura del terreno a lo largo de un eje este-oeste 


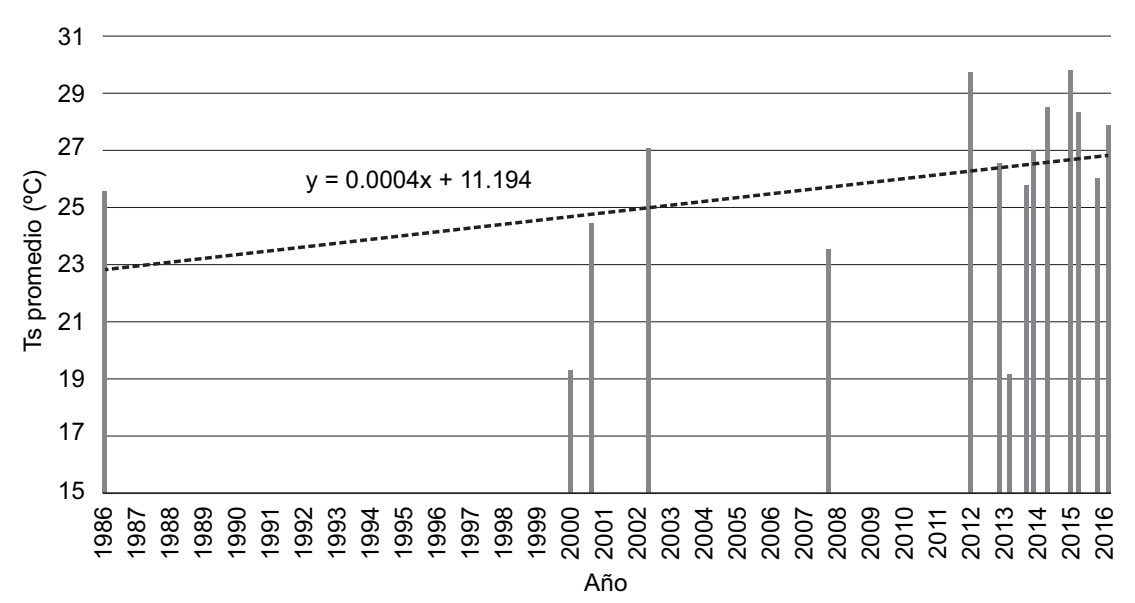

Fig. 4. Temperatura superficial promedio en el área urbana del Valle de Aburrá estimada con 15 imágenes satelitales

En lo que respecta a la distribución de densidad de vegetación, en la figura 1 se presentó un ejemplo del cálculo del indicador NDVI. En este mapa se observan las zonas verdes representativas de la ciudad, como los cerros El Volador y Nutibara (formas redondeadas oscuras en la parte central del valle junto al río Aburrá) y las áreas verdes que rodean las pistas del aeropuerto Olaya Herrera (también en la zona central del valle) junto al campo de golf El Rodeo.
El mapa de densidad de vegetación permite apreciar las zonas densamente pobladas y la correspondencia de éstas con las de mayor TS en el mapa de la izquierda en la misma figura 1. De igual manera, las áreas con mayor presencia de vegetación son las de menor TS, destacando los barrios de la comuna El Poblado en la parte inferior derecha del mapa.

En la figura 5 se presenta la densidad de vegetación a lo largo del eje este-oeste definido anteriormente,

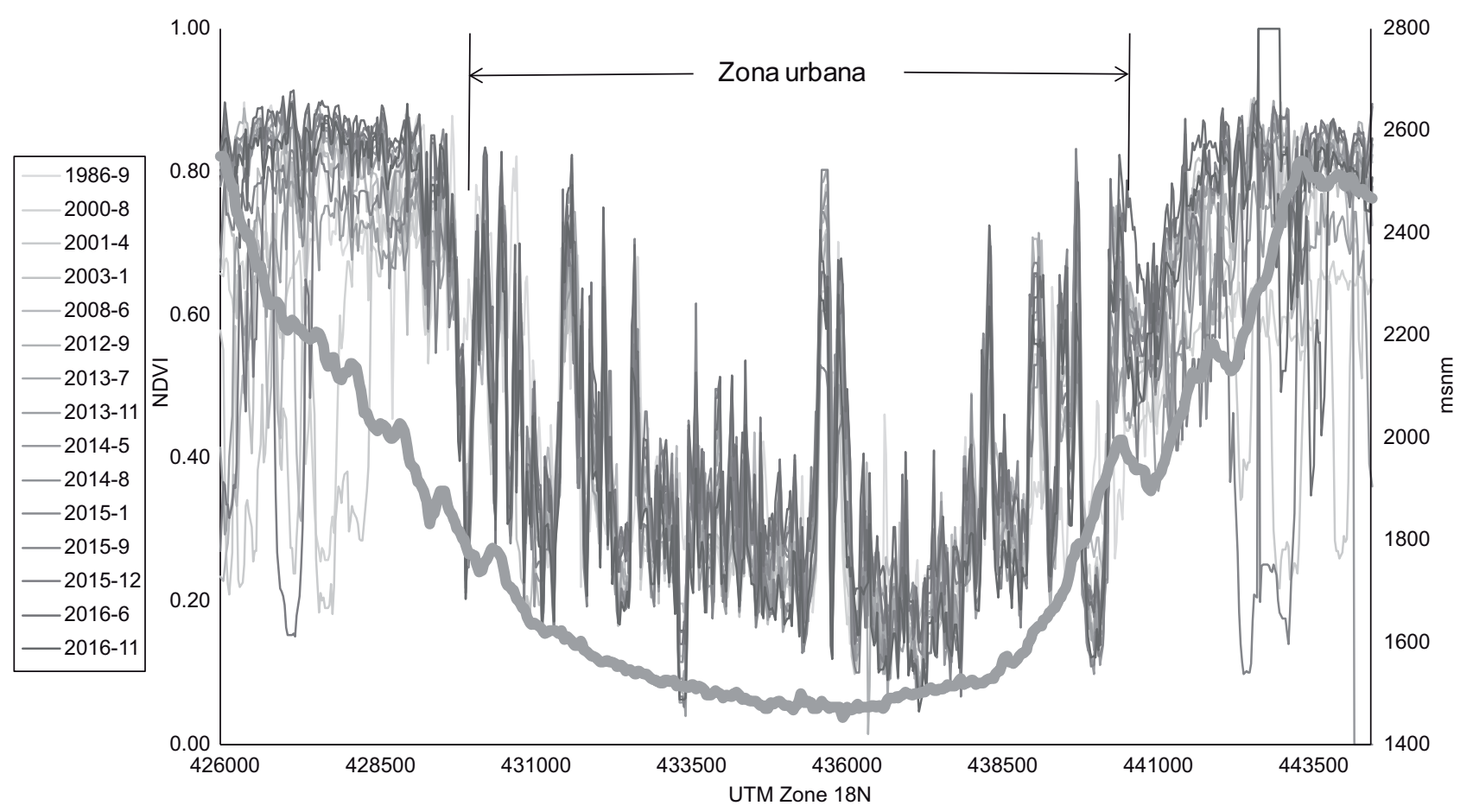

Fig. 5. Perfiles de densidad de vegetación y altura del terreno a lo largo de un eje este-oeste 


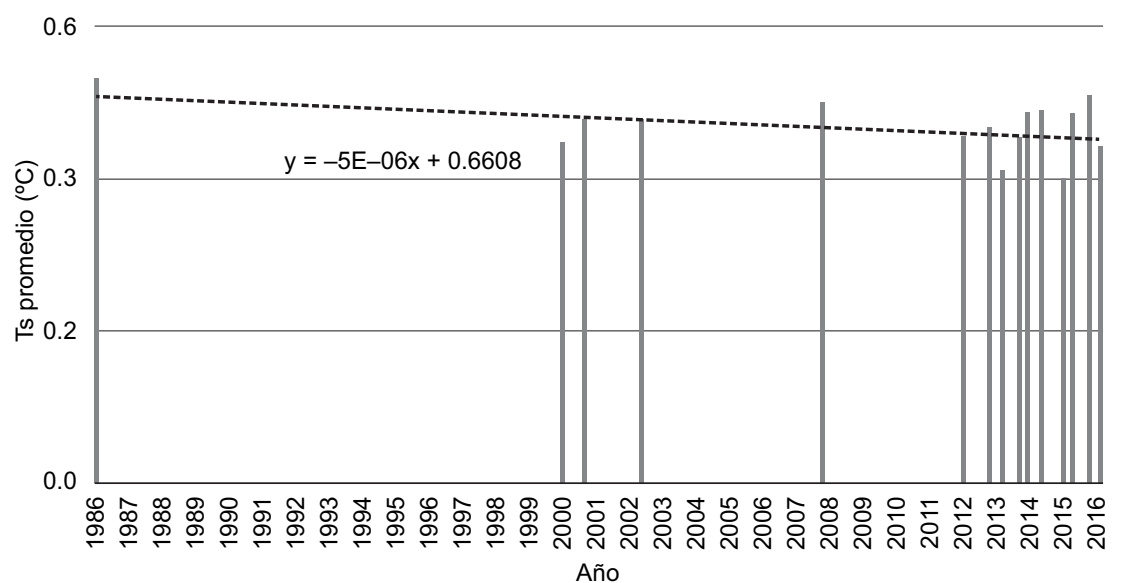

Fig. 6. Densidad de vegetación promedio en el área urbana del Valle de Aburrá estimada con 15 imágenes satelitales

para cada una de las 15 imágenes satelitales del cuadro I.

A diferencia de las estimaciones de TS, la densidad de vegetación mostró un comportamiento constante a lo largo de las 15 imágenes analizadas, sobre todo en la zona urbana. El clima templado y la abundancia de lluvias en la región, que pueden extenderse casi todo el año (febrero a junio y agosto a noviembre), generan las condiciones adecuadas para el desarrollo de la vegetación. En la figura 6 se muestra la densidad de vegetación promedio en la zona urbana para cada imagen satelital. Al igual que para la TS, la prueba de hipótesis en la recta de regresión indicó que probablemente no existe una tendencia lineal en los valores promedio de densidad de vegetación estimados $\left(t_{0}=-0.0055197<t_{\text {crit }}=\right.$ 2.17881283).

La densidad de vegetación es un indicador del desarrollo metropolitano en el Valle de Aburrá. En las partes altas del valle la densidad de vegetación tiende al valor máximo (0.8-0.9) y disminuye hacia la parte baja, donde se concentra la ocupación urbana (0.10.4). Al comparar las gráficas de NDVI (Fig. 5) y TS (Fig. 3) se distingue una relación inversa, como era de esperarse: a mayor presencia de vegetación menor temperatura superficial. Esta relación es evidente en el centro de ambas gráficas, donde se observa una disminución de TS que coincide con un incremento en la densidad de vegetación que corresponde a las zonas verdes que rodean al río Aburrá.

El AMVA se compone de más de 500 barrios pertenecientes a 10 municipios. Los mapas de la figura 7 presentan la TS promedio para cada barrio, así como la evolución de la densidad de vegetación a partir de la primera imagen analizada. La densidad de vegetación se expresa en porcentaje respecto del valor estimado para 1986.

El mapa de TS promedio permite apreciar la distribución de temperatura en el AMVA alrededor de las 10 a.m. Los barrios con mayor TS son los de la parte baja-central del valle, seguidos por los de la ladera occidental, en tanto que los de menor temperatura se encuentran en la parte alta de la ladera oriental. Considerando estos resultados, se puede afirmar que a esta hora del día la densidad constructiva no tiene un efecto notorio en la TS promedio de los barrios localizados en la parte alta de la ladera oriental del valle. En la figura 8 se muestran dos barrios de la ladera oriental. La foto de la izquierda corresponde al barrio La Cima 2, el cual se caracteriza por una alta densidad constructiva, en tanto que la foto de la derecha al barrio San Lucas, reconocido por sus construcciones de estrato alto y abundante vegetación. A pesar de que sus coberturas urbanas son distintas, ambos barrios registran la misma TS promedio a las 10 a.m. Lo anterior se debe, como se ha reiterado en este documento, a la influencia de la topografía del valle sobre la cantidad de radiación solar que llega a la superficie.

Por otra parte, el mapa de la derecha en la figura 7 permite apreciar la evolución de la densidad de vegetación tomando como punto de referencia los valores estimados con la imagen satelital de 1986. Se observa que la mayor parte del área urbana perdió entre $10 \mathrm{y}$ $35 \%$ de la densidad de vegetación. Existen zonas críticas que perdieron hasta el $83 \%$ de lo que tenían en 1986, pero también barrios que incrementaron hasta en un $58.5 \%$ su densidad vegetal. A pesar de estas diferencias a nivel de barrio, el área metropolitana, en su conjunto, parece haber mantenido estable su 

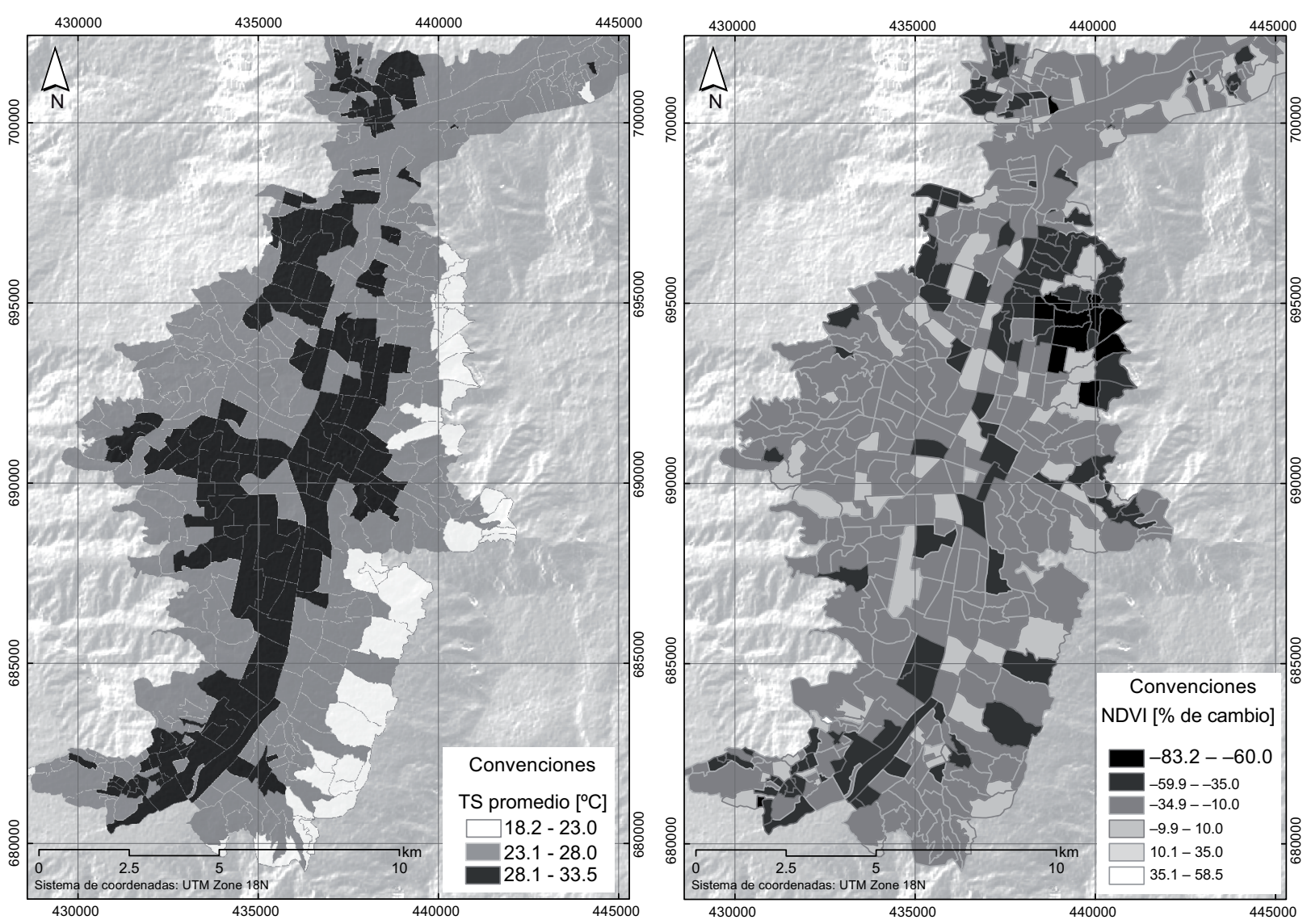

Fig. 7. Temperatura superficial promedio por barrio 1986-2016 (izquierda) y porcentaje de cambio en la densidad de vegetación respecto a 1986 (derecha)
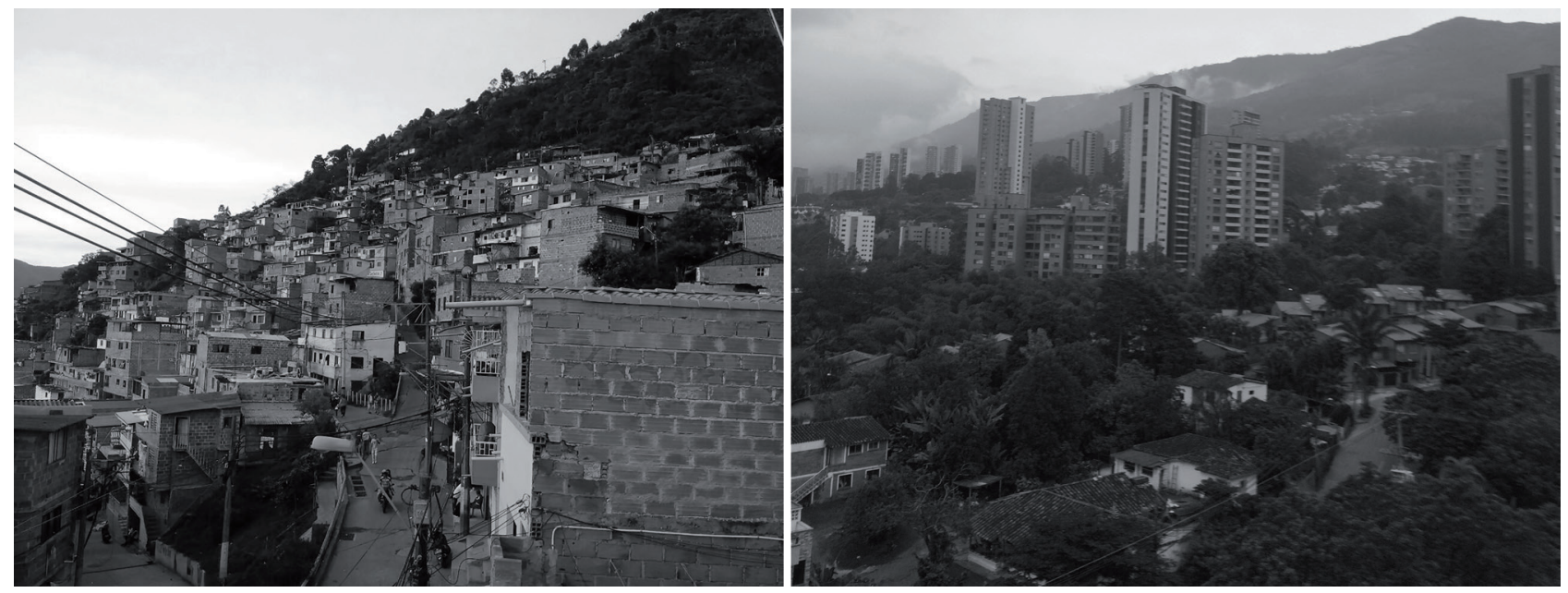

Fig. 8. Barrios La Cima 2 (izquierda) y San Lucas (derecha) (fuentes: EPM 2018 y Mosquera 2015, respectivamente) 
densidad de vegetación durante los últimos 30 años, como se observó en la figura 6.

Se considera que los barrios con las mayores pérdidas de vegetación son los que podrían experimentar incrementos de temperatura atmosférica. De acuerdo con las estimaciones realizadas, los 10 barrios con mayores porcentajes de cambio durante el periodo de análisis son: Villa Guadalupe $(-83.2 \%)$, San José La Cima (-76.5\%), Oriente (-76.3\%), La Salle $(-75.1 \%)$, Aldea Pablo VI (-72.1\%), Versalles $1(-72.1 \%)$, El Compromiso (-71.7\%), Berlín $(-70.1 \%)$, Las Granjas $(-65.1 \%)$ y Campo Valdés 2 $(-61 \%)$. Todos estos barrios pertenecen al municipio de Medellín. En la figura 7 (derecha) se puede observar que aparecen agrupados (con tono oscuro) en la parte superior derecha del mapa de densidad de vegetación.

Esta zona de la ciudad se ha caracterizado durante las últimas décadas por un crecimiento poblacional acelerado y un desarrollo urbano desordenado, el cual ha sido motivado por el alto flujo migratorio de población campesina hacia la ciudad. Robbins y Salinas (2006) estimaron que, en promedio, durante la década de 1980 y la mitad de la de 1990, a Medellín arribaron cerca de 10 mil personas anualmente. No obstante, esta cantidad aumentó a cerca de 25 mil entre 1995 y 2000 , a 35 mil entre 2000 y 2005 , para finalmente alcanzar los 50 mil después de 2005 .

\section{CONCLUSIONES}

Los resultados de esta investigación ponen de manifiesto las dificultades para estudiar el fenómeno de la ICU en regiones como el Valle de Aburrá donde, además de considerarse las variables que afectan las estimaciones de temperatura superficial - como las interferencias atmosféricas y los errores en la adquisición de la información satelital- se deben tener en cuenta los efectos asociados con el relieve del terreno a la hora en que se adquieren las imágenes Landsat y la variabilidad climática propia de la región.

A pesar de estas dificultades, en este trabajo fue posible estimar la ICS y la IIC utilizando 15 imágenes satelitales que cubren un periodo de 30 años, con lo que se logró caracterizar este fenómeno en el área metropolitana localizada en el Valle de Aburrá.

Por otro lado, el análisis de la distribución de temperatura a lo largo de un eje transversal permitió observar la IIC y cómo ésta varía a lo largo del relieve del valle y a través de los distintos momentos analizados dentro del periodo de estudio.
Por su parte, el análisis de densidad de vegetación sobre ese mismo eje mostró que la vegetación, en general, ha permanecido estable en la parte habitada del valle.

Las estimaciones de TS arrojaron valores promedio de 19.12 a $30.19^{\circ} \mathrm{C}$ en el área urbana durante el periodo 1986-2016. Esta fluctuación se atribuyó a las condiciones climatológicas cambiantes durante la adquisición de cada una de las imágenes satelitales analizadas y al crecimiento del área urbana. La IIC promedio se estimó entre 3.41 y $6.23{ }^{\circ} \mathrm{C}$, con un valor promedio general de $4.81^{\circ} \mathrm{C}$. Esta es la intensidad de la ICS en el Valle de Aburrá a las 10 a.m.

El análisis por barrio también resaltó la influencia del relieve del Valle de Aburrá en el comportamiento de la isla de calor. A las 10 a.m., los barrios de la parte alta de la ladera oriental fueron los más fríos de la ciudad $\left(21.7^{\circ} \mathrm{C}\right.$ de $\mathrm{TS}$ en promedio), mientras que los barrios de la ladera opuesta registraron temperaturas superficiales promedio de $29.6^{\circ} \mathrm{C}$ y los de la zona central de hasta $31.5^{\circ} \mathrm{C}$.

Una de las mayores tendencias de cambio a lo largo del periodo de estudio se observó en la variable densidad de vegetación. Algunos barrios mostraron una pérdida de vegetación de hasta $83 \%$ comparando los mismos meses (septiembre) de los años 1986 y 2015. Esta pérdida se debe al acelerado crecimiento urbano que ha tenido lugar en estos sitios, producto de la alta inmigración a la ciudad. Se considera que estas zonas deben priorizarse durante el diseño de la estrategia de mitigación y adaptación de la ciudad ante islas de calor.

Considerando lo anterior, se concluye que el fenómeno de ICU tiene lugar en el Valle de Aburrá y que éste se concentra en su zona central, donde existe mayor densidad urbana y menor exposición a vientos. Las acciones de mitigación y adaptación deben enfocarse a esta zona y donde se haya presentado la mayor pérdida de vegetación.

Se considera necesario analizar este fenómeno a una hora del día en que la radiación solar sea homogénea en toda el área urbana, además de valorar el aporte de distintas alternativas de mitigación y adaptación para enfrentar la ICU. Estos temas forman parte del trabajo que se desarrollará a futuro en esta línea de investigación.

\section{AGRADECIMIENTOS}

El autor desea agradecer a la Universidad EIA por el apoyo brindado para la realización de esta investigación. 


\section{REFERENCIAS}

Akbari H., Pomerantz M. y Taha H. (2001). Cool surfaces and shade trees to reduce energy use and improve air quality in urban areas. Sol. Energy 70 (3), 295-310. DOI: 10.1016/S0038-092X(00)00089-X

Aleksandrowicz O., Vuckovic M., Kiesel K. y Mahdavi A. (2017). Current trends in urban heat island mitigation research: Observations based on a comprehensive research repository. Urban Climate 21 (1),1-26. DOI: $10.1016 /$ j.uclim.2017.04.002

AMVA (2010). Atlas metropolitano del Valle de Aburrá. 1a ed. Atlas. Área Metropolitana del Valle de Aburrá, Medellín, Colombia, 46 pp.

AMVA-UNAL (2013). Aunar esfuerzos técnicos y económicos para el desarrollo e implementación de un plan de manejo integral para el arbolado urbano del Valle de Aburrá con énfasis en intervenciones para el control de la muerte súbita: Convenio 471 de 2012. Informe. Área Metropolitana del Valle de Aburrá-Universidad Nacional de Colombia, Medellín, Colombia, $37 \mathrm{pp}$.

AMVA (2017). El Valle de Aburrá. Área Metropolitana del Valle de Aburrá [en línea]. http://www.metropol. gov.co/institucional/Paginas/queeselarea.aspx 3/10/2017

Arifwidodo S. y Chandrasiri O. (2015). Urban heat island and household energy consumption in Bangkok, Thailand. Energy Procedia 79 (1), 189-194.

DOI: 10.1016/j.egypro.2015.11.461

Aristizábal E. y Yokota S. (2008). Evolución geomorfológica del Valle de Aburrá y sus implicaciones en la ocurrencia de movimientos en masa. Bol. Ciencias Tierra 24, 5-18.

Artis D.A. y Carnahan W.H. (1982). Survey of emissivity variability in thermography of urban areas. Remote Sens. Environ. 12 (4), 313-329.

DOI: 10.1016/0034-4257(82)90043-8

Asimakopoulos D., Assimakopoulos V., Chrisomallidou N., Klitsikas N., Mangold D., Michel P. y Tsangrassoulis A. (2001). Energy and climate in the urban built environment. James and James, Nueva York, EUA, $402 \mathrm{pp}$.

Bai L., Ding G., Gu S., Bi P., Su B., Qin D. y Liu Q. (2014). The effects of summer temperature and heat waves on heat-related illness in a coastal city of China, 2011-2013. Environ. Research 132 (2014), 212-219. DOI: 10.1016/j.envres.2014.04.002

Black A.L. (2013). Temperature trends and urban heat island intensity mapping of the Las Vegas Valley. Ms.C. thesis. Departament of Civil and Environmental Engineering, University of Nevada, Las Vegas, EUA, $107 \mathrm{pp}$.
Buyadi S.N.A, Wan-Mohd W.M.N y Misni A. (2014). Quantifying green space cooling effects on the urban microclimate using remote sensing and GIS techniques. Memorias. XXV International Federation of Surveyors Congress, Kuala Lumpur, Malasia, 16-21 de junio, 1-16.

Campbell-Lendrum D. y Corvalán C. (2007). Climate change and developing-country cities: Implications for environmental health and equity. J. Urban Health 84 (1), 109-115.

DOI: $10.1007 / \mathrm{s} 11524-007-9170-x$

Carlson T.N. y Ripley D.A. (1997). On the relation between NDVI, fractional vegetation cover, and leaf area index. Remote Sens. Environ. 62 (3), 241-252.

DOI: 10.1016/S0034-4257(97)00104-1

City of Toronto (2008). Urban heat island mitigation strategy toolkit [en línea]. https:/www.cip-icu.ca/Files/ Resources/STUDIO2-RYERSON-UHI-TOOLKITFINAL-REPORT.aspx 1/12/2017

Correa M., Zuluaga C., Palacio C., Pérez J. y Jiménez J. (2009). Acoplamiento de la atmósfera libre con el campo de vientos locales en una región tropical de topografía compleja. Caso de estudio: Valle de Aburrá, Antioquia, Colombia. Dyna 158, 17-27.

Corumluoglu O. y Asri I. (2015). The effect of urban heat island on Izmir's city ecosystem and climate. Environ. Sci. Pollut. R. 22 (5), 3202-3211.

DOI: $10.1007 / \mathrm{s} 11356-014-2874-\mathrm{Z}$

EPM (2018). Camino al barrio. La Cima 2 [en línea]. https://www.epm.com.co/site/home/camino-albarrio1/la-cima-2 10/5/2018

Erell E., Pearlmutter D. y Williamson T. (2011). Urban microclimate: Designing the spaces between buildings. Earthscan, Washington, DC, EUA, 266 pp.

Gartland L. (2008). Heat islands: Understanding and mitigating heat in urban areas. Earthscan, Londres, Inglaterra, $185 \mathrm{pp}$.

Gedzelman S.D., Austin S., Cermak R., Stefano N., Partridge S., Quesenberry S., y Robinson D.A. (2003). Mesoscale aspects of the urban heat island around New York City. Theor. Appl. Climatol. 75 (1-2), 29-42. DOI: $10.1007 / \mathrm{s} 00704-002-0724-2$

Harris Geospatial Solutions (2017). QUAC Background [en línea]. https://www.harrisgeospatial.com/docs/ backgroundquac.html 15/09/2017

Hermelin M. (2007). Valle de Aburrá: ¿Quo vadis? Gestión y Ambiente 10 (2), 7-16.

IDEAM (2010). Características climatológicas de ciudades principales y municipios turísticos. Instituto de Hidrología, Meteorología y Estudios Ambientales [en línea]. http://www.ideam.gov.co/documents/21021/ 21789/1Sitios+turisticos2.pdf/cd4106e9-d608-4c2991cc-16bee9151ddd 9/5/2018 
Ihara T., Kikegawa Y., Asahi K., Genchi Y. y Kondo H. (2008). Changes in year-round air temperature and annual energy consumption in office building areas by urban heat-island countermeasures and energy-saving measures. Appl. Energ. 85 (1), 12-25.

DOI: 10.1016/j.apenergy.2007.06.012

Jacobson M. (1999). Fundamentals of atmospheric modelling. 2a ed. Cambridge University Press, Nueva York, EUA, 828 pp.

Japan for Sustainability (2008). Efforts in Japan to mitigate the urban heat island effect [en línea]. https://www. japanfs.org/en/news/archives/news_id027856.html $1 / 12 / 2017$

Kieron D. y Hutchings T. (2013). Air temperature regulation by urban trees and green infrastructure [en línea]. https://www.forestry.gov.uk/pdf/FCRN012.pdf/\$file/ FCRN012.pdf 1/12/2017

Kolokothoni M., Ren X., Davies M. y Mavrogianni A. (2012). London's urban heat island: Impact on current and future energy consumption in office buildings. Energ. Buildings 47 (1), 302-311.

DOI: 10.1016/j.enbuild.2011.12.019

Landsberg H.E. (1981). The urban climate. Academic Press. Londres, Inglaterra, $275 \mathrm{pp}$.

Mosquera S. (2015). Como se vive en el barrio San Lucas de la ciudad de Medellín [en línea]. https:// suxymosquetero.wordpress.com/2015/02/05/como-sevive-en-el-barrio-san-lucas-de-la-ciudad-de-medellin/ $10 / 05 / 2018$

NASA (2006). New York City temperature and vegetation. National Aeronautics and Space Administration [en línea]. https://landsat.visibleearth.nasa.gov/view. php?id=6800 3/11/2017

NASA (2007). Landsat 7 science data user's handbook. Manual. National Aeronautics and Space Administration, Houston, EUA, 186 pp.

NASA (2012). Measuring vegetation (NDVI \& EVI). National Aeronautics and Space Administration [en línea]. http:/earthobservatory.nasa.gov/Features/ MeasuringVegetation/measuring_vegetation_2.php $10 / 07 / 2013$

NASA (2015). Vegetation limits city warming effects. National Aeronautics and Space Administration [en línea]. https://earthobservatory.nasa.gov/IOTD/view. php?id=86440 13/09/2017

NYSERDA (2006). Mitigating New York City's heat island with urban forestry, living roofs, and light surfaces. New York State Energy Research and Development Authority, Nueva York, EUA, 173 pp.

Pérez C. (1993). Los ecosistemas del Valle de Aburrá, pasado, presente y futuro. Memorias. Seminario Una Mirada al Valle de Aburrá. Medellín, Colombia, pp. 63-95.
Qaid A., Bin-Lamit H., Remaz-Ossen D., Nafida R. y Shahminan R. (2016). Urban heat island and thermal comfort conditions at micro-climate scale in a tropical planned city. Energ. Buildings 133 (1), 577-295.

DOI: 10.1016/j.enbuild.2016.10.006

Qunfang H. y Yuqi L. (2015). The effect of urban heat island on climate warming in the Yangtze River delta urban agglomeration in China. Int. J. Environ. Res. Public Health 12 (8), 8773-8789. DOI: $10.3390 /$ ijerph120808773

Robbins D.J. y Salinas D. (2006). Desempleo, inmigración y su impacto sobre el desempleo en Medellín. Análisis de las encuestas de calidad de vida-Medellín 20042005. Perfil de Coyuntura Económica 8 (1), 93-119.

Rouse J.W., Hass R.H., Schell J.A. y Deering D.W. (1974). Monitoring vegetation systems in the Great Plains with ERTS. Memorias. Third Earth Resources Technology Satellite-1 Symposium. Washington, DC, EUA. 10-14 de diciembre 1973, pp. 309-317.

Sarrat C., Lemonsu A., Masson V. y Guedalia D. (2006). Impact of urban heat island on regional atmospheric pollution. Atmos. Environ. 40 (10), 1743-1758.

DOI: 10.1016/j.atmosenv.2005.11.037

Scott M. (2006). Beating the heat. Earth Observatory, National Aeronautics and Space Administration [en línea]. http://earthobservatory.nasa.gov/Features/ GreenRoof/ 1/12/2017

Sobrino J.A., Villa R., Hurtado E., Raissouni N. y Simarro J. (1999). Aplicación de la base de datos Pathfinder al estudio del seguimiento de la dinámica de la cobertura terrestre. Memorias. VIII Congreso Nacional de Teledetección. Albacete, España. 22 al 24 de septiembre, pp. 37-40.

Sobrino J.A., Jiménez-Muñoz J.C. y Paolini L. (2004). Land surface temperature retrieval from LANDSAT TM 5. Remote Sens. Environ. 90 (4), 434-440.

DOI: $10.1016 /$ j.rse.2004.02.003

Sobrino J.A., Oltra-Carrió R., Jiménez-Muñoz J.C., Julien Y., Soria G., Franch B. y Mattar C. (2012). Emissivity mapping over urban areas using a classification-based approach: Application to the Dual-use European Security IR Experiment (DESIREX). Int. J. Appl. Earth Obs. 18,141-147.

DOI: $10.1016 /$ j.jag.2012.01.022

Susca T., Gaffin S.R. y Dell'osso G.R. (2011). Positive effects of vegetation: Urban heat island and green roofs. Environ. Pollut. 159 (8-9), 2119-26.

DOI: $10.1016 /$ j.envpol.2011.03.007

UNAL (2005). Plan de ordenación y manejo de la cuenca del río Aburrá [en línea]. http://www.metropol. gov.co/Planeacion/DocumentosAreaPlanificada/ POMCA_C2_subsistema_abiotico_RecursoAgua. pdf $10 / 05 / 2018$ 
UPM (2009). Climatología aplicada a la ingeniería y medioambiente. Universidad Politécnica de Madrid [en línea]. http://ocw.upm.es/ingenieria-agroforestal/ climatologia-aplicada-a-la-ingenieria-y-medioambiente $11 / 05 / 2018$

US-EPA (2016). Using trees and vegetation to reduce heat islands. U.S. Environmental Protection Agency [en línea]. https://www.epa.gov/heat-islands/using-treesand-vegetation-reduce-heat-islands 29/9/2017

USGS (2016). Landsat 8 (L8) Data user's handbook. Manual. U.S. Geological Survey, Dakota del Sur, EUA, 106 pp.

USGS (2017a). Landsat processing details. U.S. Geological Survey [en línea]. https://landsat.usgs.gov/landsatprocessing-details 14/09/2017

USGS (2017b). What are the band designations for the Landsat satellites? U.S. Geological Survey [en línea]. https://landsat.usgs.gov/what-are-band-designationslandsat-satellites 15/09/2017
USGS (2017c). Landsat missions. U.S. Geological Survey [en línea]. https://landsat.usgs.gov/what-are-orbitpaths-landsat-satellites 1/12/2017

USGS-EarthExplorer (2017). EarthExplorer. U.S. Geological Survey [en línea]. https://earthexplorer.usgs. gov/ 1/12/2017

Vieira-Araujo R., Albertini M., Costa-da-Silva A., Suesdek L., Soares-Franceschi N. y Marcal N. (2015). Sao Paulo urban heat islands have a higher incidence of dengue than other urban areas. Braz. J. Infect. Dis. 19 (2), 146-155. DOI: 10.1016/j.bjid.2014.10.004

Voogt J.A. (2008). Islas de calor en zonas urbanas: ciudades más calientes. Action Bioscience [en línea]. http://www.actionbioscience.org/esp/ambiente/voogt. html 1/12/2017

Watkins R. y Kolokotroni M. (2012). The London urban heat island - upwind vegetation effects on local temperaturas [en línea]. https://kar.kent.ac.uk/40220/ $28 / 11 / 2017$ 